10.1117/2.1200905.1612

\title{
Nonlinear plasmonics: controlling light with light
}

Wayne Dickson, Alexey Krasavin, Gregory Wurtz, and Anatoly Zayats

Hybridizing plasmonic structures with optically nonlinear material may enable the creation of an optical analog to the electronic circuit.

Many scientists believe that the future of signal processing and computing lies not within electronics but in the field of subwavelength optics. Controlling light at sub-wavelength scales, forbidden by traditional far-field optics, relies on surface plasmon techniques. Surface plasmon polaritons (SPPs) are electromagnetic waves that exist on the surface of metals such as gold or silver. The interaction between the electromagnetic field and the free electrons in the metal helps confine SPPs tightly to the surface. The behavior of this wave can be manipulated by nanostructuring either the surface or a nearby dielectric. By contrast, metallic particles (or nanoscale dielectric voids in a metallic host) can enhance the field by supporting localized plasmonic excitations that create superior field confinement. Both mechanisms can locally control the electromagnetic field, a pre-requisite for the development of such light-based circuitry and devices.

Researchers have used plasmonics to design and test various circuit elements such as waveguides, mirrors, lenses, and resonators based on nanostructured metal films. ${ }^{1}$ These passive plasmonic systems can provide some of the essential components for signal processing and optical circuitry, but creating optical analogs to electrical circuits requires active devices.

In order to directly make plasmonic components, scientists commonly propose two approaches, both based on the modification of the refractive index near the metal surface. The first uses the electro-optic effect exhibited, for example, by liquid crystal molecules. Here, the refractive index depends on the molecular alignment, which can be modulated using an electric field. Hybrid electro-optic devices are promising since electronic and photonic signals can be transferred, processed, and interconnected in the same metallic circuitry. A second method relies on dielectric materials with a large Kerr nonlinearity, or a refractive index that depends on the intensity of the illuminating light.
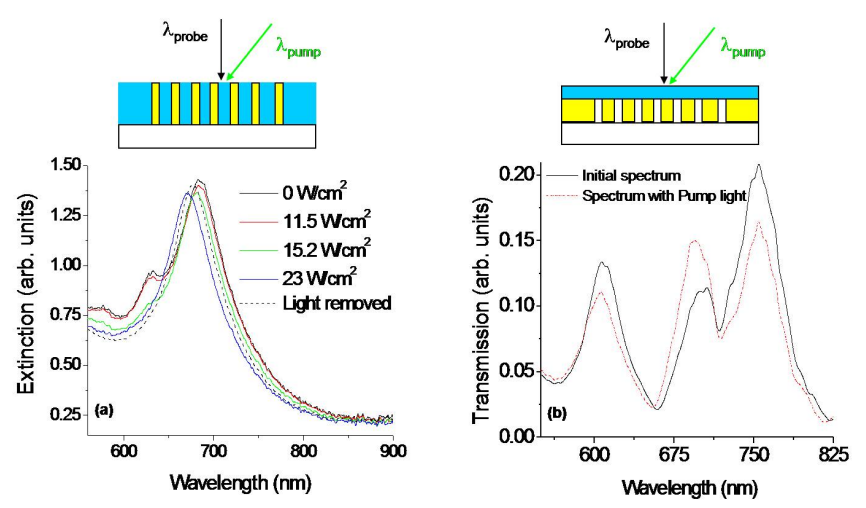

Figure 1. (left) Modulation of transmission by an array of gold nanorods (perpendicular to the substrate) and (right) a hole array in a thin gold film. Both were coated with poly-3BCMU (blue in schematic) and pumped with control light at $514 \mathrm{~nm}$.

Towards this goal, in $2006^{2,3}$ we exploited the optical nonlinearity of a poly-diacetylene molecule, 3-butoxycarbonylmethylurethane (3BCMU), on the surface plasmon polaritonic crystals (see Figure 1). These investigations followed initial work (following a similar approach) carried out as early as $2002 .{ }^{4}$ We probed the optical properties of the nanostructured array using conventional white light spectroscopy. Simultaneously, we illuminated the structure with varying intensities of pump light from a continuous wave $(\mathrm{CW})$ laser. By varying the pump power, we could control the spectral response of the structure. Surface plasmon resonances fueled the high-intensity local electromagnetic field close to the metal/dielectric boundary, enhancing the effect. In these structures, this leads to an inevitable loop, and the pump laser creates an intense field confined to the surface with a non-uniform spatial distribution. As a result, the refractive index of the polymer changes inhomogeneously, in turn changing the distribution of the field.

Because of the wavelength sensitivity of such nanostructures, slightly varying the pump wavelength (514nm to $488 \mathrm{~nm}$ ) can 


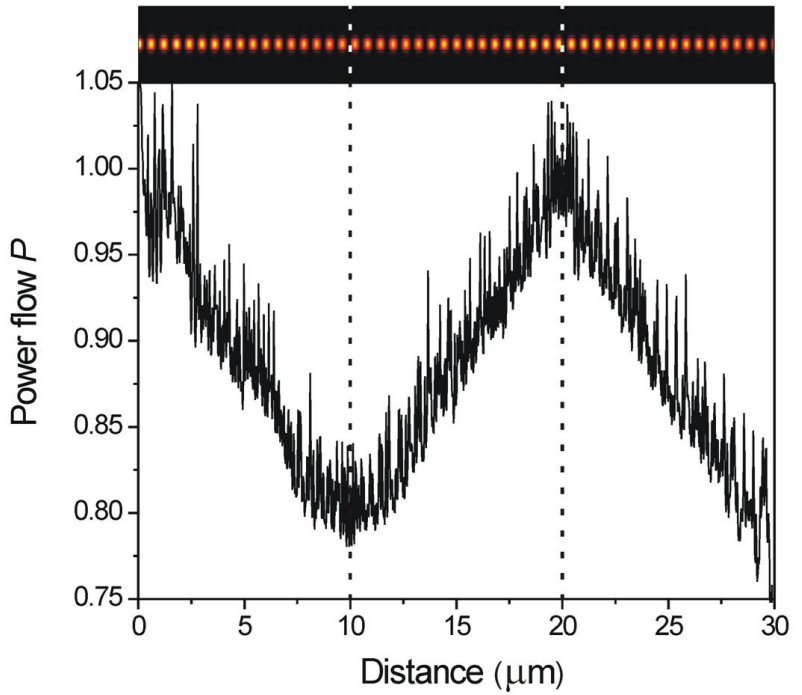

Figure 2. Dependence of the power flow, $P$, along the DLSPP waveguide that includes a section which provides gain. The modified dielectric constant of this section allows us to compensate for optical losses in the waveguide due to the metal. Modified from a prior paper. ${ }^{7}$

cause significantly different changes in optical properties. We demonstrated that such surface plasmon polaritonic crystals can also be tuned or switched electrically using liquid crystal molecules. 5

Metallic nanoparticles exhibiting a strong localized plasmon resonance, which is dependent on the size and shape of the particle, are promising elements of plasmonic systems. Chains of metallic particles are excellent candidates for waveguides, which have the advantage that a very small bend radius can be achieved without dramatically increasing losses. The field enhancement effects are ideally suited to stimulation of optical nonlinearity. We showed that arrays of vertically aligned nanowires with tunable dimensions-and hence optical properties - can be easily grown into anodic aluminium oxide templates using electrodeposition. ${ }^{6}$ The implementation of 3BCMU as the host matrix for these arrays strongly modifies the optical properties in a similar manner (see Figure 1). This suggests the feasibility of creating optically active, nonlinear metallic waveguides with such particles. ${ }^{7}$

To achieve integrated active plasmonic components for nanophotonic circuits, we proposed a concept of dielectricloaded SPP waveguides (DLSPPWs), where the photonic signal propagates in a form of highly localized SPP mode along a $600 \times 600 \mathrm{~nm}^{2}$ dielectric stripe on a surface of metal. It naturally combines high integration potential with the broadband charac- teristics of a photonic circuit. Thus, it provides the possibility of either electronic or optical control (the latter achieved through functionalizing the dielectric with non-linear materials).

We have demonstrated highly efficient passive and wavelengthselective elements based on DLSPPWs, both theoretically (see Figure 2) and experimentally. 8,9 Unfortunately, naturally available non-linear materials provide a limited refractive index change. However, highly wavelength-selective elements such as a waveguide ring resonator can enable the development of active elements. ${ }^{10}$ Using finite-element-method numerical simulations, we showed that a refractive index change of just a fraction of a percent dramatically alters signal transmission. ${ }^{11}$ The change can be introduced via electro-optic effect using an electrode on the top of the waveguide. Alternatively, we can use an SPP wave as the controlling pulse, having a resonance in the ring, leading to all-SPP active circuits. Moreover, we can modulate transmission by doping the waveguide section with active optical elements, such as quantum dots, and pumping it with an external optical source spectrally separated from the signal light. ${ }^{8}$ Further work will focus on optimizing the design and fabrication of these active elements. In addition, continuing efforts are being made to advance their integration and interaction in optical circuits.

\section{Author Information}

Wayne Dickson, Alexey Krasavin, and Anatoly Zayats

Centre for Nanostructured Media

University of Belfast, UK

Belfast, Northern Ireland

\section{Gregory Wurtz}

Department of Physics

University of North Florida

Jacksonville, Florida

\section{References}

1. A. Zayats, I. Smolyaninov, and A. Maradudin, Nano-optics of surface plasmon polaritons, Phys. Rep. 408, pp. 13-314, 2005.

2. R. P. G. Wurtz and A. Zayats, Optical bistability in nonlinear surface-plasmon polaritonic crystals, Phys. Rev. Lett. 97, p. 057402, 2006.

3. G. Wurtz and A. Zayats, Nonlinear surface plasmon polaritonic crystals, Las. Phot. Rev. 2, pp. 125-135, 2008.

4. I. Smolyaninov, A. Zayats, A. Gungor, and C. Davis, Single-photon tunneling via localized surface plasmons, Phys. Rev. Lett. 88, p. 187402, 2002.

5. W. Dickson, G. Wurtz, P. Evans, R. Pollard, and A. Zayats, Electronically controlled surface plasmon dispersion and optical transmission through metallic hole arrays using liquid crystal, Nano. Lett. 8, p. 281, 2008.

6. W. Dickson, G. Wurtz, P. Evans, D. O'Connor, R. Atkinson, R. Pollard, and A. Zayats, Dielectric-loaded plasmonic nanoantenna arrays: A metamaterial with tuneable optical properties, Phys. Rev. B 76, p. 115411, 2007.

Continued on next page 
7. W. Dickson, P. Evans, G. Wurtz, W. Hendren, R. Atkinson, R. Pollard, and A. Zayats, Towards nonlinear plasmonic devices based on metallic nanorods, J. Microsc. 229, p. 415, 2008.

8. A. Krasavin and A. Zayats, Three-dimensional numerical modeling of photonic integration with dielectric-loaded SPP waveguides, Phys.Rev. B 78, p. 045425, 2008.

9. T. Holmgaard, Z. Chen, S. Bozhevolnyi, L. Markey, A. Dereux, A. Krasavin, and A. Zayats, Dielectric-loaded plasmonic waveguide-ring resonators, Opt. Express 16, p. 13585,2008

10. T. Holmgaard, Z. Chen, S. I. Bozhevolnyi, L. Markey, A. Dereux, A. Krasavin, and A. Zayats, Wavelength selection by dielectric-loaded plasmonic components, Appl. Phys. Lett. 94, p. 051111, 2009.

11. A. Krasavin and A. Zayats, to be published. 